\title{
EXTENSIONS OF SOME FORMULAE OF A. SELBERG
}

\author{
ClaUdia A. SPIRO \\ Department of Mathematics, State University of New York \\ Buffalo, New York 14214 U.S.A. \\ (Received March 3, 1984)
}

ABSTRACT. This paper is concerned with estimating the number of positive integers up to some bound (which tends to infinity), such that they have a fixed number of prime divisors, and lie in a given arithmetic progression. We obtain estimates which are uniform in the number of prime divisors, and at the same time, in the modulus of the arithmetic progression. These estimates take the form of a fixed but arbitrary number of main terms, followed by an error term.

KEY WORDS AND PHRASES. Extensions of some formulae, formulae of A. E. ibri: prime divisors.

1980 MATHEMATICS SUBJECT CLASSIFICATION CODE. 10L20, $10 \mathrm{H} 20$.

1. INTRODUCTION.

Let $x>3$, choose $B>0$, and let $j \leq B \log \log x$ be a positive integer. In 1954, A. Selberg (cf. [1], Theorem 3) showed, for example, that the number of positive integers not exceeding $x$ which have exactly $j$ distinct prime divisors is

$$
\left.\frac{1}{(j-1) ! \log x}\left(\frac{d^{j-1}}{d z^{j-1}} \xi(z)(\log x)^{z}\right)\right|_{z=0}+0_{B}\left(\frac{x(\log \log x)^{j}}{j !(\log x)^{2}}\right),
$$

uniformly in $j$, where $\mathcal{F}(z)$ is a certain entire function. We replace $J(z)(\log x)^{z}$ by an expression of the form

$$
\sum_{\ell=1}^{M}(\log x)^{z-l_{\beta z}}
$$

and replace the error term by $0_{N}\left(x(\log \log x)^{j}(\log x)^{-M / j !)}\right.$. Moreover, we obtain results of this strength for, e.g., the number of positive integers not exceeding $x$ which lie in an arithmetic progression (say, $h \bmod k$, where $(h, k)=1$ ) and which have precisely $j$ distinct prime divisors (see Theorem 1 , below). Our results are uniform in $k$ not exceeding a fixed power of $\log x$, and in $j$. H. Delange [2] obtained comparable results for fixed k. G. J. Rieger [3] obtained related results for $M=1$, which we will discuss in greater detail in Section 7 . For a greater account of the history of the problem, see Section 4 of Norton, [5].

In Spiro [6], we apply our results to prove the following result: Let $d(n)$ denote the number of positive integers dividing the positive integer $n$, and let $M$ be any positive integer. Then there are computable constants $R_{1}, \ldots, R_{M}$ with $R_{1}>0$, such that

$$
\#\{n \leq x: d(n) \text { divides } n+1\}=x \sum_{l=1}^{M} R_{\ell}(\log x)^{\frac{1}{2}-\ell}+0_{M}\left(x(\log x)^{-M-\frac{1}{2}}\right) \text {. }
$$


2. NOTATION. THE PLAN OF THE PROOF.

Throughout this paper, $\mathrm{p}$ denotes a prime, $\mathrm{k}, \mathrm{n}$, and $\mathrm{N}$ represent positive integers, and $s$ and $z$ stand for complex numbers. We will let $\sigma$ denote the real part of $s$. The expressions $f(x) \sim g(x)$ and $f(x)=0(g(x))$ will have their usual meanings, and we will also write $f(x) \ll g(x)$ to mean that $f(x)=0(g(x))$. By either $f(x)=0_{a, b} \cdots(g(x))$ or $f(x) \ll_{a, b} \cdots g(x)$, we will signify that $f(x)=0(g(x))$, where the implied constant possibly depends on $a, b, \ldots$. Similarly, we will write $\{a, b, \ldots\}$-sufficiently large to indicate that how large is sufficient will possibly depend upon $a, b, \ldots$. When only one variable is inside the curly brackets, we will omit them, and write, e.g., a-sufficiently large. A product or sum of the form $\Pi_{p}$ or $\Sigma_{p}$ respectively denotes a product or sum over primes; thus, for example, $I_{p / n}(1+1 / p)$ denotes the product of $1+1 / p$, taken over all primes $p$ dividing $n$. Similarly, a sum of the form $\Sigma_{n \leq x}$ is assumed to extend over all positive integers $n$ not exceeding $x$. As is standard, we let $\mu(n)$ denote the Möbius function of $n$, write $\phi(n)$ for the Euler phi-function of $n$, use $\pi(x)$ for the number of primes $p \leq x$, denote the Riemann zeta-function by $\zeta(s)$ and the gammafunction by $\Gamma(z)$, designate the Dirichlet L-function of $s$ and $X$ by $L(s, X)$, and write $[x]$ for the greatest integer not exceeding the real number $x$. For simplicity, we put $\mathrm{L}_{2} \mathrm{x}=\log \log \mathrm{x}$, and $\mathrm{L}_{3} \mathrm{x}=\log \log \mathrm{x}$. Finally, $\mathrm{c}_{0}, \mathrm{c}_{1}, \ldots$ denote positive absolute constants.

Let $\mathrm{A}>0$ be constant, and let $\eta$ be a nonnegative integer-valued additive function such that

$$
\begin{array}{ll}
\eta(n) \leq A \log n & \text { for all } n, \\
\eta(p)=1 & \text { for all } p .
\end{array}
$$

Examples of functions satisfying (2.1) and (2.2) are $\omega(n)$ and $\Omega(n)$, where $\omega(n)$ is the number of distinct primes dividing $n$, and $\Omega(n)$ is the number of primes dividing $n$, counted with the multiplicities with which they occur. Also, designate by $\omega|\mu|$ the arithmetic function which takes the value $\omega(n)|\mu(n)|$ at $n$. We will let $\xi$ denote an arithmetic function which is either $\eta$ or $\omega / \mu \mid$. For any Dirichlet character $X$, and for complex numbers $s$ and $z$, set

$$
\begin{aligned}
& f(x, z, x ; \eta)=\underset{p}{\prod} \sum_{m=0}^{\infty} x\left(p^{m}\right) z^{m\left(p^{m}\right)} p_{p}^{-m s}, \\
& f(s, z, x ; \omega|\mu|)=\prod_{p}\left(1+x(p) z p^{-s}\right), \\
& g(s, z, x ; \eta)=\underset{p}{\prod}\left(\sum_{m=0}^{\infty} x\left(p^{m}\right) z^{\eta\left(p^{m}\right)} p^{-m s}\right)\left(1-x(p) p^{-s}\right)^{z}, \\
& g(s, z, x ; w|\mu|)=\underset{p}{\Pi}\left(1+x(p) z p^{-s}\right)\left(1-x(p) p^{-s}\right)^{z},
\end{aligned}
$$

wherever these products converge. Since $\omega / \mu \mid$ is not additive, this notation should cause no confusion. By the complete multiplicativity of $X$, we can expand the factor $\left(1-\chi(p) p^{-s}\right)^{z}$ by the Binomial Theorem, and formally write

$$
f(s, z, x ; \xi)=\sum_{n=1}^{\infty} a_{z}(n ; \xi) x(n) n^{-s},
$$




$$
g(s, z, X ; \xi)=\sum_{n=1}^{\infty} b_{z}(n ; \xi) x(n) n^{-s}
$$

where the coefficients $a_{z}(n ; \xi)$ and $b_{z}(n, \xi)$ are independent of $x$.

The plan of the proof will be to argue in the following manner: first, we will show that the derivation of Hilfsatz 5 of Rieger [3] can be used to estimate the sum

$$
A_{z}(x, x ; \xi)=\sum_{n \leq x} a_{z}(n ; \xi) x(n)
$$

We will establish Lemmas 3 and 4 of the next section for this purpose. If $x=\chi_{0}$ is principal, more effort will be required. The estimate made in Rieger [5] in this case relies on Hilfsatz 13 of Rieger [4]. This Hilfsatz implies that there is a constant $c_{0}>0$ such that

$$
\prod_{\mathrm{p} \mid \mathrm{k}} \frac{1-\mathrm{p}^{-\mathrm{s}}}{1-\mathrm{p}}=1+0\left(|\mathrm{~s}-1| \mathrm{L}_{2}(4 \mathrm{k})\right)
$$

for $|s-1| \log (2 k) \leq c_{0}$. In our Lemma 2 , we replace the right side by an asymptotic expansion in powers of $(s-1)$, and this expansion leads to an asymptotic expansion for $x^{-1} A_{z}\left(x, x_{0} ; \xi\right)$, descending by powers of $\log x$ (see Lemma 7, below). After we estimate $A_{z}(x, x ; \xi)$, we argue in a manner similar to that of the proof of Theorem 3 of Selberg [6], to obtain our main result.

We remark that we can obtain comparable estimates to the estimates which we obtain for the sum $A_{z}(x, x ; \xi)$ (see Lemma 7, below) for a wider class of arithmetic functions $a_{z}(n ; \xi)$ than the class given by $(2.7),(2.3),(2.4)$, and our restrictions on $\eta$. We restrict ourselves to these examples here for simplicity of exposition.

3. PRELIMINARY RESULTS.

LEMMA 1:

$$
\begin{aligned}
& \text { i) } \sum_{\mathrm{p} \mid \mathrm{k}} \mathrm{p}^{-1}(\log \mathrm{p})^{\mathrm{n}} \ll_{\mathrm{n}}\left(\mathrm{L}_{2}(3 \mathrm{k})\right)^{\mathrm{n}} \text { if } \mathrm{n} \geq 1 \text {. } \\
& \text { ii) } \sum_{\mathrm{p} \mid \mathrm{k}} \mathrm{p}^{-1} \quad \leq \mathrm{L}_{3}(8 \mathrm{k})+\mathrm{C}_{1} \text {. } \\
& \text { iii) } \underset{\mathrm{p} / \mathrm{k}}{\text { II }}\left(1-\mathrm{p}^{-1}\right)^{-1}=\frac{\mathrm{k}}{\phi(\mathrm{k})} \ll \mathrm{L}_{2}(3 \mathrm{k}) \text {. }
\end{aligned}
$$

REMARK. The special case of $i$ ) when $n=1$ is done in Rieger [4] (see equation (2.13)). The estimates $i i)$ and $i i i)$ are well known.

PROOF. The lemma is trivial if $k=1$. Otherwise, let $q$ denote the $\omega(k)^{\text {th }}$ prime. There are at most $[(\log k) / \log 2]$ primes dividing $k$, so that $q$ is at most the $[(\log k) / \log 2]^{\text {th }}$ prime. Therefore, it follows from the Chebyschev bounds for $\pi(x)$ that

$$
\mathrm{q} \ll(\log \mathrm{k}) \mathrm{L}_{2}(3 \mathrm{~K})
$$

Since $p^{-1}(\log p)^{n}$ is a decreasing function of $p$ if $p$ is sufficiently large, we can conclude that

$$
\sum_{p \mid k} p^{-1}(\log p)^{n} \ll_{n} \sum_{p \leq q} p^{-1}(\log p)^{n} .
$$


Hence, it follows from (3.4) and the Chebyschev estimate for $\pi(x)$ that

$$
\sum_{\mathrm{p} \mid \mathrm{k}} \mathrm{p}^{-1}(\log \mathrm{p})^{\mathrm{n}} \ll_{\mathrm{n}}(\log \mathrm{q})^{\mathrm{n}} \ll_{\mathrm{n}}\left(\mathrm{L}_{2}(3 \mathrm{k})\right)^{\mathrm{n}}
$$

This inequality gives $i$ ). If we repeat the argument with $n=0$, and replace our second application of the Chebyschev estimate by the estimate

$$
\sum_{p \leq q} \frac{1}{p}=L_{2}(3 q)+0(1)
$$

we obtain ii). Exponentiating ii) yields iii). //

LEMMA 2. Assume that $|s-1| \log p \leq \frac{1}{2}$ for every prime $p$ dividing $k$, and that $|s-1| \mathrm{L}_{2}(3 \mathrm{k}) \leq \mathrm{C}$, for some fixed but arbitrary positive constant $\mathrm{C}$. Then for every fixed integer $N \geq 1$, we have

$$
\mathrm{p} \mid \mathrm{k} \frac{1-\mathrm{p}^{-\mathrm{s}}}{1-\mathrm{p}^{-1}}=1+\sum_{\mathrm{n}=1}^{\mathrm{N}-1}(\mathrm{~s}-1)^{\mathrm{n}_{\mathrm{P}}} \mathrm{p}_{\mathrm{n}}(\mathrm{k})+0_{\mathrm{N}, \mathrm{C}}\left(\left(|\mathrm{s}-1| \mathrm{L}_{2}(3 \mathrm{k})\right)^{\mathrm{N}}\right) \text {, }
$$

where the coefficients $P_{n}(k)$ are real-valued, depend only on $k$ and $n$, and satisfy

$$
P_{n}(k)=0_{n}\left(\left(L_{2}(3 k)\right)^{n}\right) \text {. }
$$

PROOF. From the Taylor expansion for $e^{u}$ about $u=0$, and our first hypothesis, we can deduce that

$$
\begin{aligned}
\frac{1-p^{-s}}{1-p^{-1}} & =1+\frac{1-e^{(1-s) \log p}}{p-1} \\
& =1-\frac{1}{p-1} \sum_{j=1}^{N-1} \frac{(\log p)^{j}(1-s)^{j}}{j !}+E(s, p, N),
\end{aligned}
$$

where

$$
E(S, p, N)=0\left((|s-1| \log p)^{N} p^{-1}\right)
$$

and where

$$
\left|\frac{1}{p-1} \sum_{j=1}^{N-1} \frac{(\log p)^{j}(1-s)^{j}}{j !}+\Sigma(s, p, N)\right| \leq \frac{7}{10}
$$

By (3.9) and (3.10), we can take the logarithm of the right side of (3.8), and obtain

$$
\log \frac{1-p^{-s}}{1-p^{-1}}=\sum_{m=1}^{N-1}(s-1)^{m}(\log p)^{m} \sum_{l=1}^{m} e_{l m}(p-1)^{-l}+0_{N}\left(\frac{(|s-1| \log p)^{N}}{p}\right),
$$

for appropriate constants $e_{\ell m}$. Accordingly,

$$
\begin{aligned}
& \text { II } \frac{1-p^{-s}}{1-p^{-1}}=\exp \sum_{p / k} \log \frac{1-p^{-s}}{1-p^{-1}} \\
& =\exp \left\{\sum_{m=1}^{N-1}(s-1)^{m} \sum_{p / k} \frac{(\log p)^{m}}{p-1} Q_{m}\left((p-1)^{-1}\right)+0_{N} \sum_{p / k}\left(\frac{(|s-1| \log p)^{N}}{p}\right)\right\},
\end{aligned}
$$

where $Q_{\mathrm{m}}(\mathrm{u})=\Sigma_{\ell=1}^{\mathrm{m}} \mathrm{e}_{\ell \mathrm{m}^{\mathrm{u}}}{ }^{\ell-1}$. It now follows from Lemma 1 , part $\left.i\right)$ that the error term in $(3.12)$ is $0_{N}\left(\left(|s-1| L_{2}(3 k)\right)^{N}\right)$. Since $Q_{m}$ is a polynomial, we can also conclude from this Lemma that 


$$
\sum_{\mathrm{p} / \mathrm{k}} \frac{(\log \mathrm{p})^{\mathrm{m}}}{\mathrm{p}-1} Q_{\mathrm{m}}\left((\mathrm{p}-1)^{-1}\right) \ll_{\mathrm{m}}\left(\mathrm{L}_{3}(3 \mathrm{k})\right)^{\mathrm{m}} .
$$

If we combine this result with our second hypothesis, we find that the expression in (3.12) in curly brackets is $0_{N, C}(1)$. Consequently, we can apply the Taylor expansion of $\mathrm{e}^{\mathrm{u}}$ about $\mathrm{u}=0$ to the right side of (3.12), and the first equation of our lemma follows. To obtain the estimate for the coefficients in $P_{n}(k)$, we observe that the coefficient $R_{m}(k)$ of $(s-1)^{m}$ in (3.12) is the quantity we estimated in (3.13), and that the coefficient $P_{n}(k)$ is a finite linear combination of terms of the form $\mathrm{R}_{\mathrm{m}_{1}}(\mathrm{k}) \mathrm{R}_{\mathrm{m}_{2}}(\mathrm{k}) \ldots \mathrm{R}_{\mathrm{m}_{j}}(\mathrm{k})$ with $\mathrm{m}_{1}+\ldots+\mathrm{m}_{\mathrm{j}}=\mathrm{n}$. /

LEMMA 3. If $1>\sigma_{1}>\frac{1}{2}$ is fixed, then there is a real number $B=B\left(\sigma_{1}, \xi\right)>1$ such that the following statements hold:

i) $\sum_{n=1}^{\infty}\left|b_{z}(n ; \xi) x(n) n^{-s}\right|$

converges uniformly in $s, z$, and $X$ with $\sigma \geq \sigma_{1}$, and $|z| \leq B$.

ii) for every $\sigma_{2}>1$,

$$
\sum_{n=1}^{\infty}\left|a_{z}(n ; \xi) x(n) n^{-s}\right|
$$

converges uniformly in $s, z$, and $X$ with $\sigma \geq \sigma_{2}$, and $|z| \leq B$. If $\xi=\omega|\mu|$ or $\omega$, these results hold regardless of the choice of $B$, and $B$ does not depend on $\sigma_{1}$. If $\bar{\xi}=\Omega$, then these results are valid for $B<2 \sigma_{1}$. For $\xi \neq \omega|\mu|$, we can take any $B<\exp \left\{\left(\sigma_{1}-\frac{1}{2}\right) / A\right\}$.

REMARK. It follows from $(2.1)$ and $(2.2)$ with $n=p=2$ that $1=\eta(2) \leq A \log 2$. Consequently, we have $\exp \left\{\left(\sigma_{1}-\frac{1}{2}\right) / A\right\} \leq 2^{\sigma_{1}-\frac{1}{2}}$, so that the result for $\Omega$ is superior to the result for an arbitrary choice of $\xi$.

PROOF. First, assume that $\xi \neq \omega|\mu|$. Formally, it follows from the Binomial Theorem and (2.2) that

$$
\begin{aligned}
& \left(1-\mathrm{p}^{-\mathrm{s}}\right)^{z} \sum_{\mathrm{m}=0}^{\infty} z^{\xi\left(\mathrm{p}^{\mathrm{m}}\right)} \mathrm{p}^{-\mathrm{ms}} \\
= & 1-\frac{\mathrm{z}^{2}}{\mathrm{p}^{z \mathrm{~s}}}+\left(1+\frac{\mathrm{z}}{\mathrm{p}}\right) \sum_{\ell=2}^{\infty}(-1)^{\ell}\left(\begin{array}{l}
z \\
l
\end{array}\right) \mathrm{p}^{-\ell s}+\left(\sum_{\ell=0}^{\infty}(-1)^{\ell}\left(\begin{array}{l}
z \\
l
\end{array}\right) \mathrm{p}^{-\ell s}\right) \sum_{\mathrm{m}=2}^{\infty} \mathrm{z}^{\xi\left(\mathrm{p}^{\mathrm{m}}\right) \mathrm{p}^{-\mathrm{ms}},}
\end{aligned}
$$

for every prime $P$. Hence, from (3.16) and the definition of the coefficients $b_{z}(n ; \xi)$, we can conclude that

$$
\begin{aligned}
\sum_{j=0}^{\infty}\left|\mathrm{b}_{z}\left(\mathrm{p}^{j} ; \xi\right) \mathrm{p}^{-\mathrm{s}}\right| & \leq\left(1+\frac{z}{\mathrm{p}^{\sigma}}\right) \sum_{l=2}^{\infty}\left|\left(\begin{array}{l}
\mathrm{z} \\
l
\end{array}\right)\right| \mathrm{p}^{-\ell \sigma}+1+\frac{|\mathrm{z}|^{2}}{\mathrm{p}^{2 \sigma}}+ \\
& +\left(\sum_{l=0}^{\infty}\left|\left(\begin{array}{l}
z \\
l
\end{array}\right)\right| \mathrm{p}^{-\ell \sigma}\right) \sum_{\mathrm{m}=2}^{\infty}|\mathrm{z}|^{\xi\left(\mathrm{p}^{\mathrm{m}}\right)} \mathrm{p}^{-\mathrm{m} \sigma}
\end{aligned}
$$

Denote the sum on the left of $(3.17)$ by $E(p, z, s ; \xi)$.

Let $B$ be strictly between 1 and $\exp \left\{\left(\sigma_{1}-\frac{1}{2}\right) / A\right\}$. For $|z| \leq B$ and $\sigma \geq \sigma_{1}$, we deduce from (2.1) and (3.17) that 


$$
\begin{aligned}
& \mathrm{E}(\mathrm{p}, \mathrm{z}, \mathrm{s} ; \xi) \leq 1+\mathrm{B}^{2} \mathrm{p}^{-2 \sigma} 1+\left(1+\mathrm{Bp}^{-\sigma} 1\right) \sum_{l=2}^{\infty}\left|\left(\begin{array}{l}
z \\
l
\end{array}\right)\right|^{-l \sigma} 1+ \\
& +\left(\sum_{l=0}^{\infty}\left|\left(\begin{array}{l}
z \\
l
\end{array}\right)\right| \mathrm{p}^{-\ell \sigma_{1}}\right) \sum_{\mathrm{m}=2}^{\infty} \mathrm{p}^{\mathrm{m}\left(\mathrm{A} \log \mathrm{B}-\sigma_{1}\right)} \text {. }
\end{aligned}
$$

Now for $|z| \leq B$,

$$
\left|\left(\begin{array}{l}
z \\
l
\end{array}\right)\right| \leq-\frac{1}{\ell !} \prod_{i=1}^{l}([B]+i) \leq(l+1)^{[B]} .
$$

Hence, the last sum on $\ell$ in (3.18) is uniformly bounded in $p$ and $z$, so that (3.18) and (3.19) yield

$$
\mathrm{E}(\mathrm{p}, \mathrm{z}, \mathrm{s} ; \xi) \leq 1+\left(1+\mathrm{Bp}{ }^{-\sigma_{1}}\right)_{l=2}^{\infty} 0_{B}\left(l^{B^{\mathrm{p}}}{ }^{-l \sigma} 1\right)+0_{B}\left(\sum_{m=2}^{\infty} \mathrm{p}^{-\sigma_{2} \mathrm{~m}}\right),
$$

where $\sigma_{3}=\sigma_{1}-A \log B$. Now $\sigma_{3}>\frac{1}{2}$ by our choice of $B$, so that the final error term in $(3.20)$ is $0_{B}\left(p^{-2 \sigma} 3\right)$. Moreover,

$$
\sum_{l=2}^{\infty} \ell^{\mathrm{B}_{\mathrm{p}}}{ }^{-\ell \sigma} 1 \leq \mathrm{p}^{-2 \sigma} 1 \sum_{\ell=0}^{\infty}(l+2)^{\mathrm{B}_{2}}{ }^{-\sigma_{1} l}
$$

It follows from (3.20) and this last inequality that

$$
E(p, z, s ; \xi) \leq 1+0_{B}\left(p^{-2 \sigma} 1\right)+0_{B}\left(p^{-2 \sigma} 2\right) \text {. }
$$

In view of our notation, (3.22), and the fact that $\sigma_{1}$ and $\sigma_{3}$ both exceed $\frac{1}{2}$, we conclude that the product

$$
\text { if } \sum_{j=0}^{\infty}\left|b_{z}\left(p^{j} ; \xi\right) \times\left(p^{j}\right) p^{-s}\right|
$$

converges uniformly in $s, z$, and $x$, with $\sigma \geq \sigma_{1}$, and $|z| \leq B$. Accordingly, the series in (3.14) is uniformly bounded in $s, z$, and $X$, with $\sigma z \sigma_{1}$ and $|z| \leq B$. For fixed $B$, there is a number $\sigma_{4} \in\left(\frac{1}{2}, \sigma_{1}\right)$, such that $B<\exp \left\{\left(\sigma_{4}-\frac{1}{2}\right) / A\right\}$. By what we have just proved, the quantity in (3.14) is uniformly bounded in $s, z$, and $x$, with $\sigma \geq \sigma_{4}$ and $|z| \leq B$. But if $\sigma \geq \sigma_{1}$, and $x \geq 1$, then we have

$$
\begin{aligned}
\sum_{n>x}\left|b_{z}(n ; \xi) x(n) n^{-s}\right| & \leq \sum_{n>x}\left|b_{z}(n ; \xi)\right| n{ }^{-\sigma_{4}} \sigma_{4}-\sigma_{1} \\
& \leq x^{\sigma-\sigma_{1}} \sum_{n=1}^{\infty}\left|b_{z}(n ; \xi)\right| n{ }^{-\sigma_{4}} .
\end{aligned}
$$

Consequently, as $x$ tends to infinity, the sum on the left of (3.24) tends to zero, uniformly in $x, z$, and $x$ with $\sigma z \sigma_{1}$ and $|z| \leq B$. So, the sum in (3.14) converges uniformly in $s, z$, and $X$, as asserted. To obtain the uniform convergences of (3.15), in view of the derivation of (2.7) it is enough to show that

$$
\text { II } \sum_{\mathrm{m}=0}^{\infty}\left|x\left(\mathrm{p}^{\mathrm{m}}\right) \mathrm{z}^{\eta\left(\mathrm{p}^{\mathrm{m}}\right)} \mathrm{p}^{-\mathrm{ms}}\right|
$$

is uniformly convergent. By (2.1) and (2.2), we have 


$$
\sum_{m=0}^{\infty}\left|x\left(p^{m}\right) z^{\eta\left(p^{m}\right)} p^{-m s}\right| \leq 1+p^{-\sigma}+\sum_{m=2}^{\infty} p^{-m(\sigma-A \log B)} \text {. }
$$

By hypothesis, $\sigma_{3}=\sigma_{1}-\mathrm{A} \log \mathrm{B}>\frac{1}{2}$, and consequent $1 \mathrm{y}$

$$
\begin{aligned}
\sum_{m=0}^{\infty}\left|x\left(p^{m}\right) z^{\eta\left(p^{m}\right)} p^{-m s}\right| & \leq 1+p^{-\sigma}+p^{-2 \sigma} \sum_{m=0}^{\infty} 2^{-m \sigma} 3 \\
& =1+p^{-\sigma}+0_{B, \sigma_{1}}\left(p^{-2 \sigma_{3}}\right) .
\end{aligned}
$$

Accordingly, the quantity in (3.25) is uniformly convergent, as desired, and the lemma is proved in this case.

In the event that $\xi=\omega$, the uniform convergence of the quantity in (3.14) can be derived from (3.17). In this case, the uniform convergence of the quantity in (3.25), and hence, of the quantity in (3.15), follows from the fact that $\omega\left(p^{m}\right)=1$ whenever $\mathrm{m} \geq 1$. These derivations are similar to the argument that we have just made, and will therefore be left to the reader.

If we put $\xi=\Omega$ into (3.17), the last sum in (3.17) is the geometric series $\sum_{m=2}^{\infty}|z|^{m} p^{-m v}$. If we fix $B \in\left(1,2^{\sigma}\right)$, then this series is $o_{B}\left(p^{-2 \sigma} 1\right)$, uniformly in $z, p$, and $s$, with $\sigma \geq \sigma_{1}$, and $|z| \leq B$. The rest of the proof of the result for $\xi=\Omega$ is similar to the proof of the result for the case $\xi \neq \omega|\mu|$, given above, and will be left to the reader. Finally, suppose that $\xi=\omega|\mu|$. As in the derivation of (3.16), for every prime $p$ we have

$$
\left(1-\frac{1}{\mathrm{p}}\right)^{z}\left(1+\frac{z}{\mathrm{p}}\right)=1-\frac{z^{2}}{\mathrm{p}^{2 s}}+\left(1+\frac{z}{\mathrm{p}}\right) \sum_{\ell=2}^{\infty}\left(\begin{array}{l}
\mathrm{z} \\
l
\end{array}\right)(-1)^{\ell} \mathrm{p}^{\ell-l s} .
$$

Hence, in view of our notation, we find that

$$
\sum_{j=0}^{\infty}\left|b_{z}\left(p^{j} ; \omega|\mu|\right) p^{-s}\right| \leq 1+|z|^{2} p^{-2 \sigma} 1+\left(1+|z| p^{-\sigma} 1\right) \sum_{\ell=2}^{\infty}\left|\left(z_{\ell}^{z}\right)\right| p^{-m \sigma} 1 .
$$

Again, the remainder of the proof in this case is similar to the proof for the case in which $\xi \neq \omega|\mu|$, and we omit the details. /

For the remainder of this paper, we will assume that $1>\sigma_{1}>\frac{1}{2}$ is given, and that $B=B\left(\sigma_{1}, \xi\right)>1$ satisfies conclusions $\left.i\right)$ and $\left.i 1\right)$ of Lemma 3 . Furthermore, we will take $\sigma_{1}$ to be fixed. If a constant implied in an error term depends on $\sigma_{1}$ only inasmuch as it depends on $B$, we will indicate the dependence on $B$, but not the dependence upon $\sigma_{1}$.

LEMMA 4: For $1 \leq \mathrm{x}<\mathrm{y}$ and for $|\mathrm{z}| \leq \mathrm{B}$, there is an $\varepsilon>0$, possibly depending on $\sigma_{1}$ and $B$, such that

$$
\sum_{x<n \leq y} a_{z}(n ; \xi) x(n)=0_{B, \sigma_{1}}\left((y-x)(\log y)^{B}+y^{1-\epsilon}\right),
$$

uniformly in $z$.

PROOF: Since

$$
f(s, z, X ; \xi)=g(s, z, X ; \xi)(\zeta(s))^{z},
$$


it follows from (2.7), (2.8), and equation (1) of Rieger [3] that

$$
a_{z}(n ; \xi)=\sum_{l \mid n} d_{z}(l) b_{z}\left(\frac{n}{l} ; \xi\right),
$$

where

$$
\sum_{n=1}^{\infty} d_{z}(n) n^{-s}=(\zeta(s))^{z}
$$

We can conclude from equation (2) of Rieger [3] that $\left|d_{z}(n)\right| \leq d_{[B]+1}(n)$ for all $n$ and $z$ with $|z| \leq B$, so that (3.32) yields

$$
\left|a_{z}(n ; \xi)\right| \leq \sum_{\ell \mid n} d_{[B]+1}(n)\left|b_{z}(n ; \xi)\right| \text {. }
$$

We claim that it suffices to show that

$$
\sum_{n \leq x} \sum_{\ell \mid n} d_{[B]+1}(n)\left|b_{z}(n ; \xi)\right|=\sum_{j=1}^{[B]} k_{j, B, z} x(\log x)^{j}+0_{B, \sigma_{1}}\left(x^{1-\varepsilon}\right),
$$

for a constant $\epsilon=\epsilon\left(\sigma_{1}, B\right)>0$, and constants $\kappa_{j, B, z}$ which are uniformly bounded in $z$ with $|z| \leq B$. Indeed, from (3.34) and (3.35), we will be able to deduce that

$$
\begin{aligned}
\sum_{x<n \leq y} a_{z}(n ; \xi) x(n) \mid & \leq \sum_{y<n \leq x} \sum_{\ell \mid n} d_{[B]+1}(n)\left|b_{z}(n ; \xi)\right| \\
& =\sum_{j=0}^{[B]} k_{j, B, z}\left\{y(\log y)^{j}-x(\log x)^{j}\right\}+0_{B, \sigma_{1}}\left(y^{1-\epsilon}\right) .
\end{aligned}
$$

The Mean Value Theorem implies that

$$
y(\log y)^{j}-x(\log x)^{j}=0_{j}\left((y-x)(\log x)^{j-1}\right) \text { for } j \geq 1,
$$

and combining this fact with (3.36) verifies the claim. According to (3.34), the left side of (3.35) is

$$
\Sigma=\sum_{n \leq x}\left|b_{z}(n ; \xi)\right| \sum_{\ell \leq x / n} d_{z}(l)=\Sigma_{I}+\Sigma_{I I}+\Sigma_{I I I}
$$

where

$$
\begin{aligned}
\Sigma_{I} & =\sum_{n \leq \sqrt{x}}\left|b_{z}(n ; \xi)\right| \sum_{\ell \leq x / n} d_{z}(l), \\
\Sigma_{I I} & =\sum_{\sqrt{x}<n \leq x / 2}\left|b_{z}(n ; \xi)\right| \sum_{\ell \leq x / n} d_{z}(l), \\
\Sigma_{I I I} & =\sum_{x / 2<n \leq x}\left|b_{z}(n ; \xi)\right| .
\end{aligned}
$$

By equation (12.1.4) on p. 263 of Titchmarsh [7], we have

$$
\sum_{\ell \leq x} d_{[B]+1}(l)=\sum_{j=0}^{[B]} \lambda_{j, B} x(\log x)^{j}+0_{B}\left(x^{1-\frac{1}{[B]+1}}(\log x)^{B}\right),
$$

for certain constants $\lambda_{j, B}$.

Substituting (3.42) into (3.41) yields 


$$
\begin{aligned}
&\left|\Sigma_{I I}\right| \ll \sum_{B \sqrt{x}}<n \leq x / 2 \\
& \leq b_{z}(n ; \xi) \mid \frac{x}{n}(\log x)^{[B]} \\
& \sum_{\sqrt{x}}<n \leq x / 2 \\
&\left|b_{z}(n ; \xi)\right| \frac{x}{n}(\log x)^{[B]}\left(\frac{n}{\sqrt{x}}\right)^{1-\sigma_{1}} .
\end{aligned}
$$

Hence, we can deduce from Lemma 3, part i) that

$$
\Sigma_{I I}=0_{B}\left(x^{\left(1+\sigma_{1}\right) / 2}(\log x)^{[B]}\right) \text {. }
$$

Part i) of Lemma 3 also implies that

$$
\Sigma_{\text {III }} \leq \sum_{x / 2<n \leq x}\left|b_{z}(n ; \xi)\right|\left(\frac{x}{n}\right)^{\sigma_{1}} \underset{B}{\ll} x^{\sigma_{1}}
$$

Finally, combining (3.42) with (3.39) gives

$$
\Sigma_{I}=\sum_{n \leq \sqrt{x}} \frac{\left|b_{z}(n ; \xi)\right|}{n} \sum_{j=0}^{[B]} \lambda_{j, B} \times\left(\log \frac{x}{n}\right)^{j}+0_{B} \sum_{n \leq \sqrt{x}}\left|b_{z}(n ; \xi)\right|\left(\frac{x}{n}\right)^{1-\frac{1}{B+2}} .
$$

To bound the error term, we note that if $1-\frac{1}{B+2} \geq \sigma_{1}$, then part i) of Lemma 3 yields

$$
\sum_{n \leq \sqrt{x}}\left|b_{z}(n ; z)\right|\left(\frac{x}{n}\right)^{1-\frac{1}{B+2}}=o_{B}\left(x^{1-\frac{1}{B+2}}\right) .
$$

Otherwise, we can apply Lemma 3 , part i) to obtain

$$
\begin{aligned}
& \sum_{n \leq \sqrt{x}}\left|b_{z}(n ; \xi)\right|\left(\frac{x}{n}\right)^{1-\frac{1}{B+2}} \leq \sum_{n \leq \sqrt{x}}\left|b_{z}(n ; \xi)\right|\left(\frac{x}{n}\right)^{1-\frac{1}{B+2}}\left(\frac{\sqrt{x}}{n}\right)^{\sigma_{1}-1+\frac{1}{B+2}} \\
& =o_{B}\left(x^{\frac{1}{2}}\left(\frac{2}{B+2}+\sigma_{1}\right)\right) \text {. }
\end{aligned}
$$

It remains to estimate the main term in $(3.44)$. If we write

$$
\left(\log \frac{x}{n}\right)^{j}=(\log x)^{j}\left(1-\frac{\log n}{\log x}\right)^{j}
$$

and apply the Binomial Theorem, we find that

$$
\sum_{j=0}^{[B]} \lambda_{j, B} x\left(\log \frac{x}{n}\right)^{j}=\sum_{j=0}^{[B]} \lambda_{j, B} x(\log x)^{j} \sum_{m=0}^{j}(-1)^{m}\left(\frac{\log n}{\log x}\right)^{m}\left({ }_{m}^{j}\right) .
$$

If we make the change of variable $u=j-m$, we can rewrite this expression as

$$
\sum_{u=0}^{[B]} x(\log x)^{u} \underset{j=u}{[B]} \lambda_{j, B}(-1)^{j-u}\left({ }_{u}^{j}\right)(\log n)^{j-u} .
$$

Hence, the main term of $(3.46)$ is

$$
\sum_{u=0}^{[B]} x(\log x)^{u} \sum_{j=u}^{[B]} \lambda_{j, B}\left({ }_{u}^{j}\right) \sum_{n \leq \sqrt{x}} \frac{(-\log n)^{j-u}\left|b_{z}(n, \xi)\right|}{n} .
$$

We want to write the sum on $n$ as the sum from $n=1$ to $\infty$, plus an error term. 
It follows from part $i$ ) of Lemma 3 that this remainder term is

$$
0\left(\sum_{n \geq \sqrt{x}} \frac{\left|b_{z}(n ; \xi)\right|}{\sigma_{1}} \frac{(\log n)^{j-u}}{n-\sigma_{1}}\right)=0_{B, j, \sigma_{1}}\left((\log x)^{j-u_{x}\left(\sigma_{1}-1\right) / 2}\right),
$$

since the factor $(\log n)^{j-u} / n^{1-\sigma} 1$ is a decreasing function of $n$. Hence, we can combine whichever one of (3.47) or (3.48) applies to the error term in (3.46) with $(3.38),(3.44),(3.45),(3.52)$, and (3.53), to obtain

$$
\Sigma=\sum_{u=0}^{[B]} x(\log x)^{u} \sum_{j=u}^{[B]} \lambda_{j, B}\left({ }_{u}^{j}\right) \sum_{n=1}^{\infty} \frac{(-\log n)^{j-u}\left|b_{z}(n ; \xi)\right|}{n}+0_{B, \sigma_{1}}\left(x^{1-\varepsilon}\right),
$$

for some constant $\varepsilon=\varepsilon\left(\sigma_{1}, B\right)>0$. The claim now follows with $k_{j, B, z}$ equal to the sum on $j$ in (3.54), since part $i$ ) of Lemma 3 implies that

$$
\sum_{n=1}^{\infty} \frac{(\log n)^{j-u}\left|b_{z}(n ; \xi)\right|}{n}
$$

is uniformly bounded in $z$ for $|z| \leq B$. //

4. THE ESTIMATE FOR $A_{z}(x, x ; \xi)$.

Let $c_{2}$ be the constant $c_{6}$ from Rieger [3], put $c\left(\sigma_{1}\right)=\min \left\{c_{2}, 1-\sigma_{1}\right\}$, and assume that $0<\delta<c\left(\sigma_{1}\right)(\log (8 \mathrm{k}))^{-1}$. Cut the complex plane from 0 to $-\infty$ along the negative real axis. Then, we will define the contour $s(k, \delta)$ to be the union of the following three paths: the straight line segment from $1-c\left(\sigma_{1}\right)(\log (8 \mathrm{k}))^{-1}$ to 1 - $\delta$ along the negative real axis below the cut; the circle of radius $\delta$ and center 1 , traversed once counterclockwise, beginning and ending at the point 1 - $\delta$ (but not including this point); the straight line segment from $1-\delta$ to $1-c\left(\sigma_{1}\right)\left(\log (8 k)^{-1}\right.$ along the negative real axis above the cut.

LEMMA 5. Let $k<e^{\sqrt{\log x}}$, and let $x$ be a character modulo $k$. The following results are uniform in $z$ and $k$ with $|z| \leq B$ :

i) If $x$ is nonreal, then $A_{z}(x, x ; \xi)=0_{B}\left(x e^{-c_{3} \sqrt{\log x}}\right)$.

ii) If $x$ is real but nonprincipal, then we have

$$
A_{z}(x, x ; \xi)=0_{B}\left(x^{-c_{3} \sqrt{\log x}}\right)+0_{B}\left(\frac{x^{\beta(k)}}{\phi(k)}(\log 2 k)^{c 4^{B}}\right) \text {, }
$$

where $\beta(k)=\max \left\{\sigma: L\left(\sigma, \lambda_{2}\right)=0\right\}$.

iii) If $x=x_{0}$ is principal, then we have

where

$$
A_{z}(x, x ; \bar{s})=L_{z}(x, k)+0_{B}\left(x e^{-c_{3} \sqrt{\log x}}\right)
$$

$$
L_{z}(x, k)=\frac{1}{2 \pi 1} \int_{S(k, \delta)} f\left(x, z, x_{0} ; \xi\right) \frac{x^{s}}{s} d s .
$$

PROOF. As we indicated at the end of Section 2 , we replace $(L(s, X))^{z}$ by 


$$
f(s, z, X ; \xi)=g(s, z, x ; \xi)(L(s, x))^{z}
$$

in the proof of Hilfsatz 5 of Rieger [3]. To insure that Lemma 3 is applicable to $g\left(s, z, x_{;} \xi\right)$ on the paths of integration occurring in the proof of Hilfsatz 5 , we replace the constant $c_{6}$ in the equation following (6) of Rieger [3] by our constant $c\left(\sigma_{1}\right)$. Now part $\left.i\right)$ of Lemma 3 implies that $g(s, z, x ; \xi)$ is uniformly bounded in $\sigma \geq \sigma_{1}$ and $|z| \leq B$, so that $g(s, z, X ; \xi)=0_{B}(1)$ on each of these paths of integration. Hence, (4.4) yields

$$
|f(s, z, x ; \xi)| \ll_{B} \mid\left(\left.L(s, x)\right|^{z} \mid .\right.
$$

Furthermore, in place of the estimate

$$
\sum_{x<n \leq y} d_{z}(n) x(n)=0_{B}\left((y-x)(\log y)^{B}+y^{1-\frac{1}{B+2}}\right)
$$

uniformly for $|z| \leq B$ and for $1 \leq x<y$ (see line 19 on $p .185$ of Rieger [3]), we have Lemma 4. We leave the details to the reader. //

$$
\text { Set } Q(k)=\underset{p \mid k}{\Pi} p \text {. }
$$

LEMMA 6. Choose $u>0$, assume that $k<\exp \sqrt{\log x}$ and that $Q(k) \leq(\log x)^{u}$, let $X$ be a nonprincipal character modulo $k$, and suppose that $|z| \leq B$. Then

$$
A_{z}(x, x ; \xi)=O_{B, u}\left(x e^{-c 3^{\sqrt{\log x}}}\right),
$$

uniformly in $k$ and $z$.

PROOF. If $X$ is nonreal, the result follows from Lemma 5, part 1 ), a fortior 1 . If $X$ is real, then we can conclude from Hilfsatz 1 of Walfisz [8] that $X$ is also a character modulo $Q$, for at least one element $Q$ of $\{Q(k), 2 Q(k), 4 Q(K)\}$. According to a theorem of C. L. Siegel (cf. Estermann [2], Theorem 48), if $\varepsilon>0$ is given, and $n$ is $\varepsilon$-sufficiently large, then $\beta(n)$ is less than $1-(n / 4)^{-\varepsilon}$. Consequently, if $Q(k)$ is $\varepsilon$-sufficiently large, we can deduce from Lemma 5, part i1), with $k$ replaced by $Q$, that

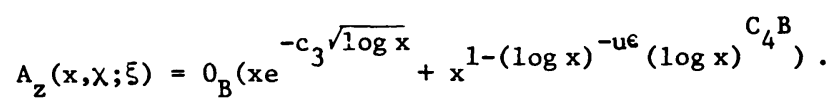

Thus, we can take $\epsilon=1 /(3 u)$ to obtain the desired result. Now there are only finitely many values of $k$ for which $Q(k)$ fails to be $\epsilon$-sufficiently large for $\epsilon=1 /(3 u)$. In each such case, $\beta(Q(k)), \beta(2 Q(k))$, and $B(4 Q(k))$ are all less than 1 , so that our lemma also follows from part ii) of Lemma 5 . /I

LEMMA 7. Select $u>0$, assume that $k<\exp \sqrt{\log x}$ and that $Q(k) \leq(\log x)^{u}$, and suppose that $|z| \leq B$. Let $X_{0}$ denote the principal character modulo $k$, and let $\mathrm{N}$ be a fixed but arbitrary positive integer. Then we have

$$
\begin{aligned}
A_{z}\left(x, X_{0} ; \tilde{\zeta}\right)= & x \sum_{\ell=1}^{N} \beta_{\ell z}(k)(\log x)^{z-\ell} \\
& +0_{B, N, u}\left(x(\log x)^{\operatorname{Re~} z-N-1}\left(L_{2}(3 k)\right)^{N}\left(\frac{\phi(k)}{k}\right)^{\operatorname{Re~} z}\right)
\end{aligned}
$$

uniformly in $k, N$, and $z$, where 


$$
\beta_{\ell z}(k)=\left.\frac{1}{(l-1) ! \Gamma(z-l+1)}\left(\frac{\partial^{l-1}}{\partial_{s}{ }^{l-1}}\left\{\frac{(s-1)^{z} f\left(x, z, x_{0} ; \xi\right)}{s}\right\}\right)\right|_{s=1} .
$$

Furthermore,

$$
\left|\Gamma(z-\ell+1) \beta_{\ell z}(k)\right| \ll_{\ell, B}\left(L_{2}(3 k)\right)^{\ell}
$$

uniformly in $k$ and $z$.

PROOF. First, we rewrite $(4.4)$ as

$$
L_{z}(x, k)=\frac{x}{2 \pi i} \int_{S(k, \delta)} x^{s-1}(s-1)^{-z}\left\{\frac{(s-1)^{z} f\left(s, z, x_{0} ; \xi\right)}{s}\right\} d s \text {. }
$$

By (4.5),

$$
\frac{(s-1)^{z} f\left(s, z, x_{0} ; \xi\right)}{s}=h(x, z, k ; \xi)\left(\prod_{p \mid k} \frac{1-p^{-s}}{1-p^{-1}}\right)^{z}
$$

where

$$
h(s, z, k ; \zeta)=s^{-1} g\left(s, z, x_{0} ; \xi\right)((s-1) \zeta(s))^{z} \prod_{p \mid k}\left(1-p^{-1}\right)^{z} .
$$

We can conclude from (3.8), (3.10), and Lemma 2 that

$$
\prod_{p \mid k} \frac{1-p^{-s}}{1-p^{-1}}-1=\sum_{n=1}^{N-1}(s-1)^{n_{p_{n}}}(k)+0_{N}\left(\left(|s-1| L_{2}(3 k)\right)^{N}\right)
$$

has magnitude less than $7 / 10$, where

$$
P_{n}(k)=0_{n}\left(\left(L_{2}(3 k)\right)^{n}\right)
$$

depends only on $k$ and $n$. Hence, the Binomial Theorem Implies ihat

$$
\begin{aligned}
& \left(\prod_{p \mid k} \frac{1-p^{-s}}{1-p^{-1}}\right)^{z}=\sum_{j=0}^{N-1}\left(\begin{array}{l}
z \\
j
\end{array}\right)\left(\sum_{n=1}^{N-1^{*}}(s-1)^{n_{P_{n}}}(k)+0_{n}\left(\left(|s-1| L_{2}(3 k)\right)^{N+1}\right)\right)^{j} \\
& +0_{B, N}\left(|s-1|^{N}\left(L_{2}(3 k)\right)^{N}\right) \\
& =1+\sum_{j=1}^{N-1} R_{j z}(Q)(s-1)^{j}+0_{B, N}\left(\left(|s-1| L_{2}(3 k)\right)^{N}\right) \text {, }
\end{aligned}
$$

uniformly in $z$ and $k$, where the coefficients $R_{j z}(k)$ of $(s-1)^{j}$ satisfy

$$
R_{j z}(k)=O_{B, j}\left(\left(L_{2}(3 k)\right)^{j}\right)
$$

Now $(s-1) \zeta(s)$ is analytic and bounded away from zero on $\{s:|s-1| s 1 / 2\}$. Furthermore, part $i)$ of Lemma 3 implies that $g(s, z, x ; \xi)=0_{B}(1)$ uniformly in $k$, $s$, and $z$ with $|z| \leq B$ and $\sigma \geq \sigma_{1}$, and that $g(s, z, x ; \xi)$ is analytic in $\sigma>\sigma_{1}$ provided that $|z| \leq B$. Hence, it follows from (4.15) and Cauchy's inequality for the coefficients of a power series that

$$
h(s, z, k ; \xi)=\sum_{j=0}^{\infty} T_{z, j}(k)(s-1)^{j},
$$


with coefficients $T_{z, j}(k)$ which satisfy

$$
\left|\mathrm{T}_{z, j}(\mathrm{k})\right| \ll_{B, \sigma_{1}}\left(1-\sigma_{1}\right)^{-j}\left|\underset{p \mid k}{\pi}\left(1-p^{-1}\right)^{z}\right|=\left(\frac{1}{1-\sigma}\right)^{j}\left(\frac{\phi(k)}{k}\right)^{\operatorname{Re} z} .
$$

Thus,

$$
\left|\sum_{j=N}^{\infty} T_{z, j}(k)(s-1)^{j}\right| \ll_{B, \sigma_{1}}\left(\frac{\phi(k)}{k}\right)^{\operatorname{Rez}} \sum_{j=N}^{\infty}\left(\frac{|s-1|}{1-\sigma_{1}}\right)^{j} \ll_{B, \sigma_{1}}\left(\frac{\phi(k)}{k}\right)^{\operatorname{Rez}}|s-1|^{N} \text {, }
$$

uniformly in $k, s$, and $z$ with $|z| \leq B$ and $|s-1| \leq \frac{1}{2}\left(1-\sigma_{1}\right)$. By construction, any point $s$ along the path of integration $s(k, \delta)$ satisfies $|s-1| \leq \frac{1}{2}\left(1-\sigma_{1}\right)$, so that

$$
h(s, z, k ; \xi)=\sum_{j=0}^{N-1} T_{z, j}(k)(s-1)^{j}+0_{B, \sigma_{1}}\left(|s-1|^{N+1}\left(\frac{\phi(k)}{k}\right)^{R e z}\right)
$$

along this path. So, we can conclude from $(4.14),(4.18),(4.19),(4.21)$, and $(4.23)$ that

$$
\frac{(s-1)^{z} f\left(s, z, x_{0} ; \xi\right)}{s}=\sum_{j=0}^{N-1} \alpha_{j z}(k)(s-1)^{j}+0_{B, \sigma_{1}, N}\left(\left(|s-1| L_{2}(3 Q)\right)^{N}\left(\frac{\phi(k)}{k}\right)^{R e z}\right),
$$

where the coefficients $\alpha_{j z}(k)$ satisfy

$$
\mid \alpha_{j z}(k) \alpha \ll_{B, \sigma_{1}, j}\left(L_{2}(3 Q)\right)^{j}\left(\frac{\phi(k)}{k}\right)^{\operatorname{Re} z} .
$$

Since power series representations are unique, we must have

$$
\alpha_{j z}(k)=\left.\frac{1}{j !}\left(\frac{\partial^{j}}{\partial s^{j}}\left\{\frac{(s-1)^{z} f\left(x, z, x_{0} ; \xi\right)}{s}\right\}\right)\right|_{s=1} .
$$

We can conclude from (4.4) and (4.24) that

$$
\begin{aligned}
L_{z}(x, k) & =\sum_{j=0}^{N-1} \alpha_{j z}(k) x I(x, z-j, k)+ \\
& +0_{B, \sigma_{1}, N}\left(x\left(L_{2}(3 Q)\right)^{N}\left(\frac{\phi(k)}{k}\right)^{R e} \int_{S(k, \delta)}\left|(s-1)^{N-z}\right|^{\sigma-1}|d s|\right),
\end{aligned}
$$

where

$$
I(x, w, k)=\frac{1}{2 \pi i} \int_{S(k, \delta)}(s-1)^{-w_{x} s-1} d s .
$$

Let $c(0)$ denote the contour which goes from $-\infty$ to $c\left(\sigma_{1}\right)$ along the negative real axis below the cut, traverses $s(k, \delta)$, and then returns to $-\infty$ along the negative real axis, above the cut. Since $k<\sqrt{\log x}$,

$$
I(x, w, k)=\int_{C(0)}(s-1)^{-w_{x} s-1} d s+0_{B, \sigma_{1}, N}\left(e^{-c_{5} \sqrt{\log x}}\right),
$$

uniformly in $w$ and $k$ with $|w| \leq B+N$. If we let $-(s-1) \log x=u$, and recall the Hankel integral representation for the gamma-function (cf. the last equation on p. 245 of Whittacker and Watson [10]), we find that 


$$
I(x, w, k)=\frac{1}{\Gamma(w)}(\log x)^{w-1}+0_{B, \sigma_{1}, N}\left(e^{-c_{5} \sqrt{\log x}}\right) .
$$

To bound the remainder term in (4.27), we separate $s(k, \delta)$ into the circular part $\mathrm{K}(\delta)$ and the union $\mathrm{H}(\mathrm{k}, \delta)$ of the two horizontal strips of $\mathrm{S}(\mathrm{k}, \delta)$. Choose $\delta=(\log x)^{-1}$. Then

$$
\int_{k(\delta)}\left|(s-1)^{N-2}\right| x^{\sigma-1}|d s| \ll(\log x)^{\operatorname{Re} z-N-1}
$$

Now, it certainly suffices to prove this lemma when $\mathrm{N}>\mathrm{B}$. In that case, we have

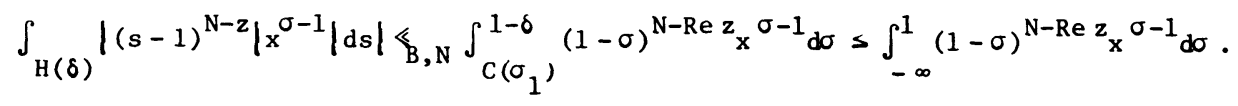

Upon making the change of variable $t=(1-\sigma) \log x$, we arrive at

$$
\begin{aligned}
\int_{H(\delta)}\left|(s-1)^{N-z}\right| x^{\sigma-1}|d s| & \ll_{B, N}|\Gamma(N-\operatorname{Re} z)|(\log x)^{\operatorname{Re} z-N-1} \\
& \ll_{B, N}(\log x)^{\operatorname{Re~} z-N-1} .
\end{aligned}
$$

Thus, the error term in $(4.27)$ is

$$
0_{B, \sigma_{1}, N}\left(\left(L_{2}(3 Q)\right)^{N}\left(\frac{\phi(k)}{k}\right)^{\operatorname{Re} z} \times(\log x)^{\operatorname{Re} z-N-1} .\right.
$$

Furthermore, by $(4.15)$, the main term in $(4.27)$ is

$$
\sum_{j=0}^{N-1} \frac{1}{\Gamma(z-j)} \alpha_{j z}(k) x(\log x)^{z-j}+0_{B, \sigma_{1}, N}\left(x^{-c_{5} \sqrt{\log x}}\right) .
$$

The lemma now follows from (4.26), (4.27), and part iii) of Lemma 5. //

5. THE STATEMENT AND PROOF OF THE MAIN RESULT

Let $h, j$, and $k$ be integers with $j \geq 0, k \geq 1$, and with $(h, k)=1$. Set

$$
\begin{aligned}
& \pi_{j}(x, k, h ; \xi)=\{\{n x: \xi(n)=j, n \equiv h(\bmod k)\}, \\
& \pi_{j}(x, x ; \xi)= \sum x(n) \\
& n \leq x, \xi(n)=j
\end{aligned}
$$

THEOREM 1. Choose $\mathrm{u}>0$, and let $\mathrm{N}$ be a fixed but arbitrary positive integer. Let $h, j, k$ be integers with $1 \leq j<\mathrm{BL}_{2} \mathrm{x},(h, k)=1,1 \leq k<\exp \sqrt{\log x}$, and $Q(k) \leq(\log x)^{u}$. Then we have

$$
\begin{aligned}
\pi_{j}(x, k, h ; \xi)=\frac{1}{\phi(k)} & \left.\frac{x}{(j-1) !}\left(\frac{\partial^{j-1}}{\partial z^{j-1}} \sum_{\ell=1}^{N} z^{-1} \beta_{l z}(k)(\log x)^{z-\ell}\right)\right|_{z=0}+ \\
& +0_{B, N, u} \frac{1}{\phi(k)}\left(\frac{x\left(L_{2} x\right)^{j}}{j !}(\log x)^{-N-1}\left(L_{2}(3 Q(k))\right)^{N}\right)
\end{aligned}
$$

uniformly in $h, j$, and $k$, where the coefficients $\beta_{l z}(k)$ are given by (4.11).

PROOF. In view of our notation, the orthogonality relations for characters yield

$$
\pi_{j}(x, k, h ; \xi)=\frac{1}{\phi(k)} \sum_{x \bmod k} \overline{\chi(h)} \pi_{j}(x, x ; \xi) \text {. }
$$


Since any character $\chi$ modulo $k$ is completely multiplicative, $\eta(n)$ is additive, and $\omega / \mu j(n)$ is additive when restricted to squarefree $n$, it follows from (2.3), (2.4), (2.7), and our definition of $A_{z}(x, x ; \xi)$ that

$$
A_{z}(x, x ; \xi)=\sum_{j=0}^{\infty} \pi_{j}(x, x ; \xi) z^{j}
$$

This sum is actually finite. If $C$ is any circle of radius less than $B$, with center 0 , traversed once counterclockwise, then Cauchy's Residue Theorem implies that

$$
\pi_{j}(x, x ; \xi)=\frac{1}{2 \pi i} \int_{C} A_{z}(x, x ; \xi) z^{-j-1} d z \text {. }
$$

Now after the proof of Lemma 3, we declared that $B$ would exceed 1 for the remainder of this paper. So, for nonprincipal $X$, we can let $C$ have radius 1 and apply Lemma 6 to obtain

$$
\pi_{j}(x, x ; \bar{\zeta})=0_{B, u}\left(x e^{-c_{3} \sqrt{\log x}}\right) .
$$

If $x=X_{0}$ is principal, then $X_{0}$ is also the principal character modulo $Q(k)$. Hence, we can estimate $A_{z}(x, X ; \xi)$ by Lemma 7, with $k$ replaced by $Q(k)$. In (4.11), we can view $X_{0}$ as either the principal character modulo $k$ or modulo $Q(k)$, so that $e_{\ell z}(k)=e_{\ell z}(Q(k))$. Thus, if we put $Q=Q(k)$, we have

$$
\begin{aligned}
\pi_{j}\left(x, X_{0} ; \xi\right) & =x \sum_{\ell=1}^{N} \frac{1}{2 \pi i} \int_{C} z^{-1} \beta \beta_{\ell z}(k)(\log x)^{z-\ell} z^{-j} d z+ \\
& +0_{b, N, u}\left(x(\log x)^{-N-1}\left(L_{2}(3 Q)\right)^{N} \int_{C}\left|z^{-j-1}\right|\left(\frac{\phi(0)}{Q} \log x\right)^{R e z}|d z| .\right.
\end{aligned}
$$

By (4.12), $z^{-1} \beta_{\ell z}(k)$ is analytic at $z=0$, and consequently Cauchy's Residue Theorem yields

$\frac{1}{2 \pi i} \int_{C^{-1}} z^{-1} \beta_{\ell z}(k)(\log x)^{z-\ell} z^{-j} d z+\left.\frac{1}{(j-1) !}\left(\frac{\partial^{j-1}}{\partial z^{j-1}}\left\{z^{-1} \beta_{\ell z}(k)(\log x)^{z-\ell}\right\}\right)\right|_{z=0}$.

If we substitute (5.8) into (5.7), and then substitute (5.6) and (5.7) into (5.3), we find that it suffices to prove that the remainder term in (5.7) is

$$
O_{B, N, \sigma_{1}}\left(\frac{x\left(L_{2} x\right)^{j}}{j !}(\log x)^{-N-1}\left(L_{2}(3 Q)\right)^{N}\right) \text {. }
$$

Since $\mathrm{j}<\mathrm{BL}_{2} \mathrm{x}$, we can let the circle $C$ in (5.7) have radius $j / \mathrm{L}_{2} \mathrm{x}$. Thus, the integral in the error term is

$$
I=2 \frac{\left(L_{2} x\right)^{j}}{j^{j}}\left(\int_{0}^{\pi} \exp \left\{(j \cos \theta)\left(L_{2} x\right)^{-1}\left(L_{2}\left(\frac{\phi(Q)}{Q} \log x\right)\right\} d \theta\right) .\right.
$$

Since $Q \leq k<\exp \sqrt{\log x}$, we can conclude from part iii) of Lemma 1 that

$$
0<\mathrm{L}_{2}\left(\frac{\phi(\mathrm{Q})}{\mathrm{Q}} \log \mathrm{x}\right) \leq \mathrm{L}_{2} \mathrm{x}
$$

Hence, since $\cos \theta \geq 0$ for $0 \leq \theta \leq \pi / 2$ and $\cos \theta \leq 0$ for $\pi / 2 \leq \theta \leq \pi$, we can 
bound the integrand by 1 for $\pi / 2 \leq \theta \leq \pi$, and by $\epsilon^{j \cos \theta}$ for $0 \leq \theta \leq \pi / 2$. Thus,

$$
I \ll\left(L_{2} x\right)^{j} j^{-j}\left(\int_{0}^{\tau / / 2} e^{j \cos \theta} d \theta+1\right) .
$$

It is well known that $\int_{0}^{\pi / 2} e^{j \cos \theta} d \theta=0\left(e^{j} j^{-1 / 2}\right)$ (in fact, we have $\int_{0}^{\pi} e^{j \cos \theta} d \theta=\pi I_{0}(j)$, where $I_{0}$ is the 0 th Bessel function of purely imaginary argument). If we combine this result with (5.12), and apply stirling's formula for $j$ !, we find that

$$
I \ll\left(L 2^{x}\right)^{j} e^{j}(j !)^{-1}
$$

Since $I$ is the integral in the error term in (5.7), that error is (5.9), and the theorem follows. //

REMARK. Upon performing the differentiation indicated in our theorem, we find that we can replace the main term of our estimate for $\pi_{j}(x, k, h ; \xi)$ by

$$
\sum_{\ell=1}^{N}(\log x)^{-\ell_{j}} j, l, k\left(L_{2} x\right)
$$

Where $J_{j, l, k}(u)$ is a polynomial in $u$ of degree $j-1$.

REMARK. From Lemma 3, we can deduce that Theorem 1 is valid for the functions $\xi=\omega / u \mid$ and $\xi=\omega$ for any value of $B>1$, and that Theorem 1 holds for $\xi=\Omega$, provided that $1<\mathrm{B}<2$. Furthermore, in view of the statement of Theorem 1 , the requirement that $B$ exceed 1 can be replaced by the assumption that $B$ be positive. 6. AN ANALOG OF THE SATHE-SELBERG FORMULAE

THEOREM 2. Fix $B^{\prime}$ with $0<B^{\prime}<B$. Select $u>0$, and let $h, j$, $k$ be integers with $1 \leq j \leq B^{\prime} L_{2} x,(h, k)=1,1 \leq k<\exp \sqrt{\log x}$, and $Q(k) \leq(\log x)^{u}$. Then we have

$$
\begin{aligned}
& \pi_{j}(x, k, h ; \xi)=\frac{x}{\phi(k) \log x} \frac{\left(L_{2} x\right)^{j-1}}{(j-1) !}\left[\frac{g\left(1, \frac{j-1}{L_{2} x}, x_{0} ; \xi\right)}{\Gamma\left(1+(j-1) / L_{2} x\right)}\left(\frac{\phi(k)}{k}\right)^{\frac{j-1}{L_{2} x}}+\right. \\
& \left.+0_{B, B^{\prime}, u}\left(\frac{j}{\left(L_{2} x\right)^{2}}\left(L_{3}(16 k)\right)^{2}\right)\right] \text {, }
\end{aligned}
$$

uniformly in $h, j$, and $k$, where $x_{0}$ denotes the principal character modulo $k$.

REMARK. According to Lemma 3, we can take $B$ to be large enough so that our theorem is valid for any choice of $B^{\prime}>0$ if $\xi=\omega / \mu /$ or $\omega$; for any selection of $B^{\prime}$ with $0<B^{\prime}<2$ if $\omega=\Omega$; and for any choice of $B^{\prime}$ satisfying

$0<\mathrm{B}^{\prime}<\exp \left\{\left(\sigma_{1}-\frac{1}{2}\right) / \mathrm{A}\right\}$ provided that $\xi \neq \omega|\mu|$. Here, $\mathrm{A}$ is the constant mentioned in (1).

PROOF. We can conclude from (44) and Theorem 1 with $\mathrm{N}=1$, that

$$
\begin{aligned}
\pi_{j}(x, k, h ; \xi)=\frac{1}{\phi(k)} \frac{x}{(j-1) !} & \left.\left(\frac{\partial^{j-1}}{\partial z^{j-1}}\left\{z^{-1} \beta_{1 z}(k)(\log x)^{z-1}\right\}\right)\right|_{z=0}+ \\
& +0_{B}\left(\frac{1}{\gamma(k)} \frac{x\left(L_{2} x\right)^{j}}{j !}(\log x)^{-2} L_{2}(3 Q(k))\right),
\end{aligned}
$$


where

$$
\beta_{1 z}(k)=\frac{1}{\Gamma(z)}\left(\frac{\phi(k)}{k}\right)^{z} g\left(1, z, x_{0} ; \xi\right) .
$$

By the functional equation for the gamma-function, we have

$$
\beta_{1 z}(k)=\frac{1}{\Gamma(z+1)}\left(\frac{\phi(k)}{k}\right)^{z} g\left(1, z, x_{0} ; \xi\right) \text {. }
$$

For $j=1$, the theorem follows from (6.2) and (6.4). If $j>1$, set

$$
\lambda(z)=\lambda(z, k ; \xi)=\beta_{1 z}(k) \text {. }
$$

Since part $i)$ of Lemma 3 implies that $g\left(1, z, x_{0} ; \xi\right)$ is bounded uniformly in $z$ with $|z|<B$, and in $k$, and since $B^{\prime}<B$, we can deduce from Cauchy's inequality for the derivative of an analytic function that $g^{\prime}\left(1, z_{0}, x_{0} ; \xi\right)$ and $g^{\prime \prime}\left(1, z, x_{0} ; \xi\right)$ are bounded uniformly in $z$ and $k$ with $|z| \leq B^{\prime}$. Furthermore, $1 / \Gamma(z+1)$ is entire, so that its first two derivatives are uniformly bounded in $z$ with $|z| \leq B^{\prime}$ Hence, it follows from (6.5), (6.4), and part iii) of Lemma 1 that

$$
\left|\lambda^{\prime \prime}(z)\right| \ll_{B_{,}, B^{\prime}}\left(\frac{\delta(k)}{k}\right)^{\operatorname{Re} z}(\log (3 k / \phi(k)))^{2} \ll_{B, B^{\prime}}\left(\frac{\phi(k)}{k}\right)^{\operatorname{Re} z}\left(L_{3}(16 k)\right)^{2},
$$

uniformly in $k$ and $z$ with $|z| \leq B^{\prime}$. From (6.2), (6.5), and Cauchy's Residue Theorem, we can conclude that

$$
\begin{aligned}
& \pi_{j}(x, k, h ; \xi)=\frac{1}{\phi(k)} \frac{x}{\log x} \frac{1}{2 \pi i} \int_{\mathcal{C}^{0}} \lambda(z)(\log x)^{z} d z+ \\
& \quad+0_{B, u}\left(\frac{1}{\phi(k)} \frac{x\left(L_{2} x\right)^{j}}{j !} \frac{L_{2}(3 Q(K))}{(\log x)^{2}}\right),
\end{aligned}
$$

where $C$ denotes the contour $|z|=(j-1) / L_{2} x$, traversed once counterclockwise. Put $r=(j-1) / L_{2} x$. Then the integral in (6.7) is

$$
\frac{\lambda(r)}{2 \pi i} \int_{C}(\log x)^{z} z^{-j} d z+\frac{1}{2 \pi i} \int_{C}\{\lambda(z)-\lambda(r)-\lambda(r)(z-r)\}(\log x)^{z} z^{-j} d z
$$

If $\overline{r z}$ denotes the straight line segment from $r$ to $z$, then we have

$$
\lambda(z)-\lambda(r)-(z-r) \lambda^{\prime}(r)=\int_{\frac{r z}{}}(z-w) h^{\prime \prime}(w) d w,
$$

for $|z| \leq B^{\prime}$. So, it follows from (6.6) and the Triangle Inequality that

$$
\left|\lambda(z)-\lambda(r)-(z-r) \lambda^{\prime}(r)\right| \ll_{B, B^{\prime}}\left(L_{3}(16 k)\right)^{2}\left(\frac{\phi(k)}{k}\right)^{\operatorname{Re} z}|r-z|^{2},
$$

again uniformly in $k$ and $z$ with $|z| \leq B^{\prime}$. We now apply this estimate to the second integral in (6.8). At the same time, we evaluate the first integral in (6.8) by Cauchy's Residue Theorem. Since the quantity in (6.8) is the integral in (6.7), we obtain

$$
\begin{aligned}
\int_{C} \lambda(z)(\log x)^{z} d z & =\frac{\left(L_{2} x\right)^{j-1}}{(j-1) !} \lambda(r)+ \\
& +0_{B, B}\left(\left(L_{3}(16 K)\right)^{2} \int_{C}|r-z|^{2}\left(\frac{\phi(k)}{k} 10 g x\right)^{\operatorname{Re} z}|z|^{-j}|d z|\right) .
\end{aligned}
$$


By our choice of $C$, we can rewrite the integral in (6.11) as

$$
4 r^{3-j} \int_{0}^{\pi}(1-\cos \theta) e^{(j-1) \cos \theta}\left(\frac{k}{\phi(k)}\right)-r \cos \theta d \theta
$$

On the interval $[0,1 / \sqrt{10 j}]$, the integrand is $0\left(j^{-1} e^{j}\right)$. For $1 / \sqrt{10 j} \leq \theta \leq \pi / 2$, the integrand is at most $(\sin \theta) / \sin (1 / \sqrt{10 j})$. Finally, by part $i i i)$ of Lemma 1 , the integrand is $\left.0\left(\mathrm{~L}_{2}(8 \mathrm{k})\right)^{\mathrm{r}}\right)$ on the interval $[\pi / 2, \pi]$. Thus, the integral in (6.11) is

$$
4 \mathrm{r}^{3-\mathrm{j}_{0}} 0\left(\mathrm{e}^{\mathrm{j}} \mathrm{j}^{-3 / 2}\right)+0\left(\exp \left\{(\mathrm{j}-1)\left(\mathrm{L}_{2}(8 \mathrm{k})\right) / \mathrm{L}_{2} \mathrm{x}\right\}\right)
$$

We will show that the second error in (6.13) can be absorbed into the first one. Indeed, if $j-1 \leq\left(L_{2} x\right) / L_{3} x$, then

$$
\left.\exp \left\{(j-1) L_{2}(8 k)\right) / L_{2} x\right\} \leq e \ll e^{j-1} j^{-3 / 2},
$$

since $k \leq x$. Furthermore, if $j-1>\left(L_{2} x\right) / L_{3} x$, then

$$
\left.\exp \left\{(j-1) L_{2}(8 k)\right) / L_{2} x\right\} \leq \exp \left\{B_{2}(8 k)\right\} \ll_{u}\left(L_{2} x\right)^{B} \ll_{B, u} e^{j-1} j^{-3 / 2},
$$

since $k \leq(\log x)^{u}$. In either case, we have shown that the integral in $(6.11)$ is $0_{B, u}\left(r^{3-j} e^{j-3 / 2}\right)$. Therefore, if we substitute (6.11) into (6.7), we find that $\pi_{j}(x, k, h ; \xi)=\frac{1}{\phi(k)} \frac{x}{\log x} \frac{\left(L_{2} x\right)^{j-1}}{(j-1) !} \lambda(r)+0_{B, u}\left(\frac{1}{\phi(k)} \frac{x\left(L_{2} x\right)^{j}}{j !} \frac{L_{2}(3 Q(k))}{(\log x)^{2}}\right)+$

$$
+0_{B, B^{\prime}, u}\left(\frac{x}{\phi(k) \log x} r^{3-j} e^{\left.j j^{-3 / 2}\left(L_{3}(16 k)\right)^{2}\right)}\right. \text {. }
$$

By applying Stirling's formula for $j$ ! to the second error term, using the fact that $Q(k) \leq(\log x)^{u}$ to estimate the first error term, and recalling $(6.4),(6.5)$, and the definition of $r$, we can now deduce the asserted result. //

7. AN APPLICATION TO THE STRENGTHENING OF SOME RESULTS OF G. J. RIEGER

By using Lemmas 6 and 7, we can improve Satze 2 through 7 of Rieger [3] by replacing each estimate by $x$ times an asymptotic expansion descending by powers of $\log \mathrm{x}$. We illustrate the idea with an example:

THEOREM 3. (cf. Satz 2 of Rieger [3]): Select $u>0$, suppose that $\mathrm{k}<\exp \sqrt{\log \mathrm{x}}$ and that $\mathrm{Q}(\mathrm{k}) \leq(\log \mathrm{x})^{\mathrm{u}}$, and assume that $|\mathrm{z}| \leq \mathrm{B}$. Let $\ell$ be any integer relatively prime to $\mathrm{k}$, and let $\mathrm{N}$ be a fixed but arbitrary positive integer. Then we have

$$
\begin{aligned}
& \sum_{n \leq x} \quad a_{z}(n ; \xi)=\frac{x}{\phi(k)} \sum_{l=1}^{N} \beta_{l z}(k)(\log x)^{z-l}+ \\
& \mathrm{n} \equiv \mathcal{L}(\bmod \mathrm{k}) \\
& +0_{B, N, u}\left(\frac{x}{\phi(k)}(\log x)^{\operatorname{Re} z-N-1}\left(L_{2}(3 k)\right)^{N}\left(\frac{\phi(k)}{k}\right)^{\operatorname{Re} z}\right)
\end{aligned}
$$

uniformly in $k, \ell, N$, and $z$, where the coefficients $\beta_{\ell z}(k)$ are given by (4.11) and satisfy $(4.12)$. 
PROOF. According to the orthogonality relations for characters, we have

$$
\begin{aligned}
& \sum_{n=x} a_{z}(n ; \xi)=\frac{1}{\phi(k)} \quad \sum_{x \bmod k} \overline{\chi(l)} A_{z}(x, x ; \xi) . \\
& n \equiv l(\bmod k)
\end{aligned}
$$

This theorem is now an immediate consequence of Lemmas 6 and 7. //

Furthermore, if we replace $f(s, z, X ; \xi)$ by $(L(s, X))^{z}$, we can argue as in Lemma 5 to obtain an estimate for $\sum_{n \leq x} d_{z}(n) x(n)$ of the same form as the expression on the right side of (4.10). Then, we can reason as in the proof of Theorem 3 , but using Hilfsatz 5 of Rieger [3] in place of Lemma 6, to arrive at the following result:

THEOREM 4. (cf. Satz 1 of Rieger [3]): Under the hypotheses of Theorem 3, we have

$$
\begin{aligned}
& \sum_{n \leq x} \quad d_{z}(n)=\frac{x}{\phi(k)} \sum_{l=1}^{N} \gamma_{l z}(k)(\log x)^{z-l}+ \\
& \mathrm{n} \equiv \ell(\bmod k) \\
& +0_{B, N, u}\left(\frac{x}{\phi(k)}(\log x)^{\operatorname{Re} z-N-1}\left(L_{2}(3 k)\right)^{N}\left(\frac{\phi(k)}{k} ; \operatorname{Re~} z\right)\right.
\end{aligned}
$$

uniformly in $k, \ell, N$, and $z$, where the coefficients $\gamma_{\ell z}(k)$ are given by

$$
\gamma_{\ell z}(k)=\left.\frac{1}{(l-1) ! \Gamma(z-\ell+1)}\left(\frac{\partial^{\ell-1}}{\partial s^{\ell-1}}\left\{\frac{(s-1)^{z}\left(L\left(s, x_{0}\right)\right)^{z}}{s}\right\}\right)\right|_{s=1} \text {. }
$$

Furthermore,

$$
\left|z \gamma_{\ell z}(k)\right| \ll_{\ell, B}\left(L_{2}(3 k)\right)^{\ell}
$$

uniformly in $k$ and $z$.

ACKNOWLEDGEMENT. The author would like to thank G. J. Rieger for enquiring about what improvements could be made in Rieger [3] by using the results of this paper. Finally, we gratefully acknowledge the help of a referee, who made a number of helpful comments in regard to our work.

\section{REFERENCES}

1. SELBERG, A. Note on a paper by L. G. Sathe, J. Indian Math. Soc. (N.S.) 18 (1954), $83-87$.

2. DELANGE, H.L. Sur des Formules de Atle Selberg, Acta Arth. 19 (1971), $105-146$ (errata insert).

3. RIEGER, G.J. Zum Teilerproblem von Atle Selberg, Math. Nachr. 30 (1965), 181-192.

4. RIEGER, G.J. Uber die Anzahl der als Summe von zwei Quadraten darstellbaren und in einer primen Restklasse gelegenen Zahlen unterhalb einer positiven Schranke. II, J. reine angew. Math. 217 (1965), 200-216.

5. NORTON, K.K. On the Number of Restricted Prime Factors of an Integer, II. Acta Math. 143 (1979), 9-38.

6. SPIRO, C.A. How of ten does the Number of Divisors of an Integer Divide its Successor?, J. London Math. Soc. (to appear).

7. TITCHMARSH, E. The Theory of the Riemann Zeta-Function, Oxford, at the Clarendon Press, 1951. $346 \mathrm{pp}$.

8. WALFISZ, A. Zur additiven Zahlentheorie. II, Math Zeit. 43 (1936), 592-607. 
9. ESTERMAN, T. Introduction to Modern Prime Number Theory, Cambridge Tracts in Mathematics and Mathematical Physics, no. 41, Cambridge, at the University Press, 1952. $x+75$ pp.

10. WHITTAKER, E.T. and WATSON, G.N. A Course of Modern Analysis, Cambridge University Press, fourth ed. reprinted, 1963; original fourth ed., 1927, 608 pp. 


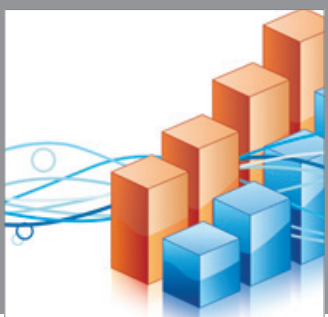

Advances in

Operations Research

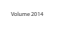

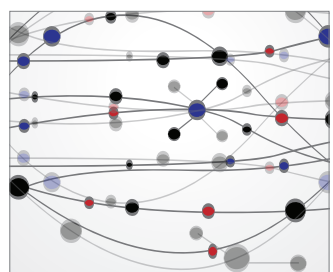

\section{The Scientific} World Journal
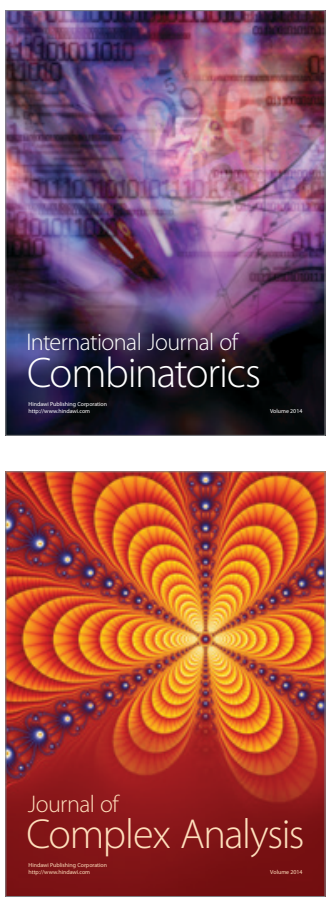

International Journal of

Mathematics and

Mathematical

Sciences
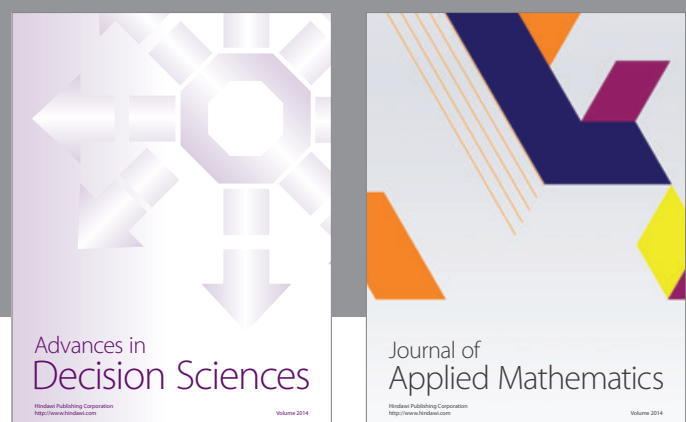

Journal of

Applied Mathematics
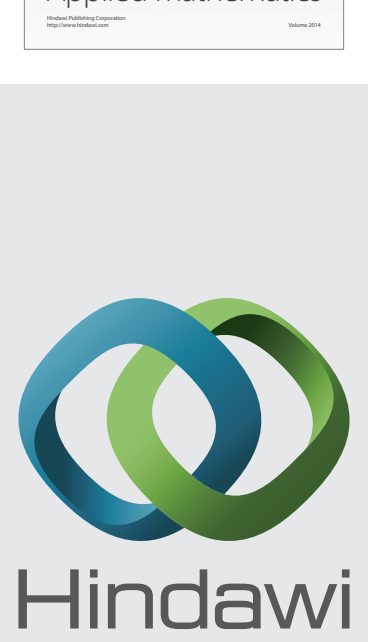

Submit your manuscripts at http://www.hindawi.com
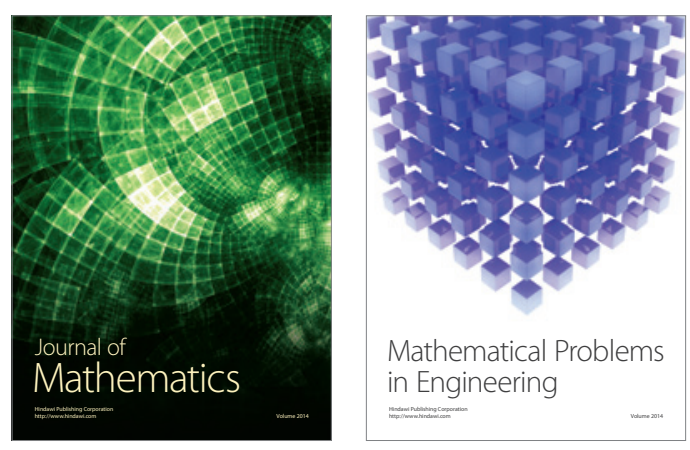

Mathematical Problems in Engineering
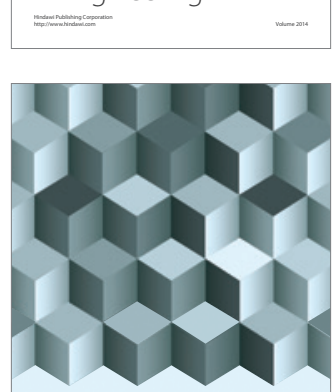

Journal of

Function Spaces
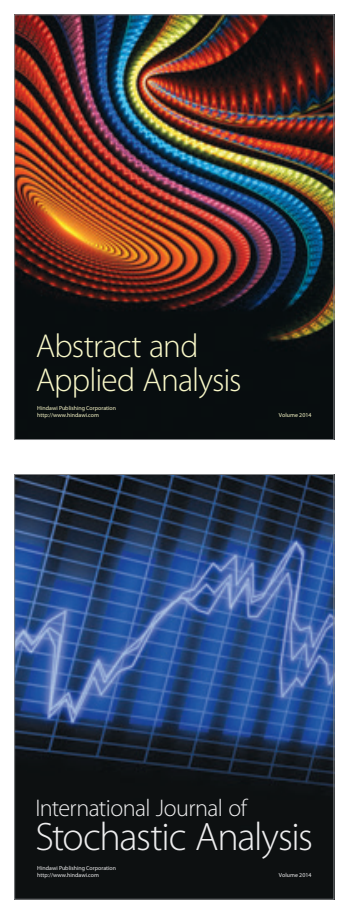

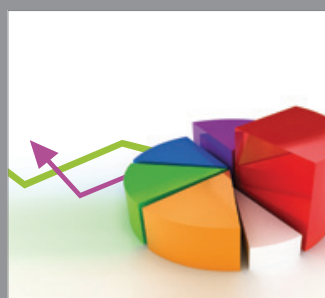

ournal of

Probability and Statistics

Promensencen
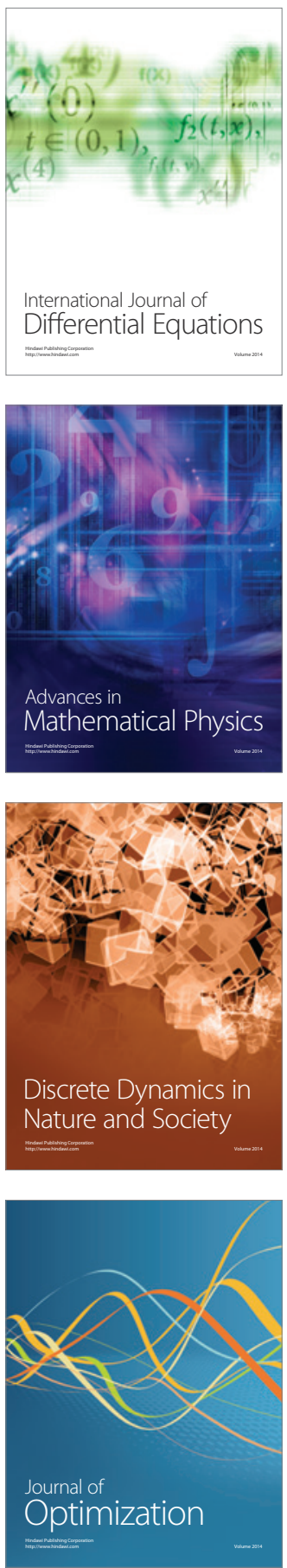